Table 1 Mean VAS Scores and \% of clinical management decision attributed to inflammation, damage, and distress in patients with rheumatic diseases

\begin{tabular}{|c|c|c|c|c|c|}
\hline Mean VAS Scores & $\begin{array}{l}A L L \\
N=570\end{array}$ & $\begin{array}{l}R A \\
N=98\end{array}$ & $\begin{array}{l}O A \\
N=1311\end{array}$ & $\begin{array}{l}F M \\
N=89\end{array}$ & $\mathbf{P}^{*}$ \\
\hline VAS DOCGL & $\begin{array}{l}4.4 \\
(1.6)\end{array}$ & $4.6(1.8)$ & $4.4(1.5)$ & $\begin{array}{l}5.2 \\
(1.6)\end{array}$ & 0.04 \\
\hline VAS DOCINF & $\begin{array}{l}1.8 \\
(2.0)\end{array}$ & $2.8(2.4)$ & $0.7(1.1)$ & $\begin{array}{l}0.8 \\
(1.3)\end{array}$ & $<0.001$ \\
\hline VAS DOCDAM & $\begin{array}{l}3.1 \\
(2.2)\end{array}$ & $3.8(2.3)$ & $4.4(1.8)$ & $\begin{array}{l}1.7 \\
(1.9)\end{array}$ & $<0.001$ \\
\hline VAS DOCSTR & $\begin{array}{l}2.1 \\
(2.9)\end{array}$ & $1.2(2.2)$ & $1.5(2.5)$ & $\begin{array}{l}6.0 \\
(2.5)\end{array}$ & $<0.001$ \\
\hline $\begin{array}{l}\mathrm{p} \text { (DOCINF vs } \\
\text { DOCDAM) }\end{array}$ & 0.001 & 0.01 & $<0.001$ & $<0.001$ & \\
\hline $\begin{array}{l}\mathrm{p} \text { (DOCINF vs } \\
\text { DOCSTR) }\end{array}$ & 0.11 & $<0.001 \dagger$ & $<0.001$ & $<0.001$ & \\
\hline \multicolumn{6}{|c|}{ Mean $\%$ of clinical management decision attributed to. } \\
\hline$\%$ inflammation & $29(31)$ & $39(29)$ & $12(19)$ & $6(11)$ & $<0.001$ \\
\hline$\%$ damage & $48(35)$ & $52(30)$ & $73(31)$ & $18(23)$ & $<0.001$ \\
\hline$\%$ distress & $22(34)$ & $9(20)$ & $15(27)$ & 76 (27) & $<0.001$ \\
\hline
\end{tabular}

*ANOVA - RA vs OA vs FM † -only comparison in which DOCINF higher than DOCSTR

Conclusions: VAS scores and estimates of the proportions of DOCGL attributed to damage were higher than for inflammation in all groups, including RA patients as a group. Control of inflammation remains a primary concern for rheumatologists, but has improved considerably in recent years, and damage and distress may have become more prominent in routine care. Systematic quantitation of inflammation, damage, and distress, in addition to DOCGL, appears feasible and of value in routine clinical care.

Disclosure of Interest: T. Pincus Shareholder of: Dr. Pincus holds a copyright and trademark for MDHAQ and RAPID3 for which he receives royalties and license fees. All revenue is used to support further development of quantitative questionnaire measures for patients and doctors in clinical rheumatology care., I. Castrejon: None declared, J. Block: None declared

DOI: 10.1136/annrheumdis-2018-eular.5588

\section{FRI0679 HIGH SENSITIVITY CARDIAC TROPONIN T IN PSORIATIC ARTHRITIS PATIENTS: A CROSS- SECTIONAL CONTROLLED STUDY}

V. Furer ${ }^{1}$, S. Shenhar-Tsarfaty ${ }^{2}$, S. Berliner ${ }^{2}$, U. Arad ${ }^{1}$, D. Paran ${ }^{1}$, O. Rogowski ${ }^{2}$, I. Shapira' ${ }^{2}$, H. Matz ${ }^{3}$, O. Elkayam ${ }^{1} .{ }^{1}$ Rheumatology, ${ }^{2}$ Internal Medicine,

${ }^{3}$ Dermatology, Tel Aviv Medical Center, Tel Aviv, Israel

Background: High sensitivity cardiac troponin (hs-cTnT) is a biomarker for cardiovascular disease (CVD) in the general population. (1) Psoriatic arthritis (PsA) confers an increased risk for CVD (2) but there are no biological markers to stratify CVD risk in PsA patients.

Objectives: To determine the level of serum hs-cTnT in a PsA cohort and controls without inflammatory disease, and further characterize the PsA cohort with detectable hs-cTnT.

Methods: Serum hs-cTnT level was measured with a sandwich immunoassay method in a consecutive PsA cohort $(n=96)$. The control cohort was based on apparently healthy individuals recruited during routine annual health examination, Tel-Aviv Medical Center Inflammatory Survey (TAMCIS) $(n=6,052)$. hs-cTnT was measured in carefully matched controls $(n=88)$, manually selected from the TAM$\mathrm{CIS}$, based on gender, age, BMI, hypertension, and hyperlipidemia prevalence. $\mathrm{Hs}-\mathrm{cTnT} \geq 5 \mathrm{ng} / \mathrm{L}$ was used as a cutoff for the detectable level. Multiple regression analysis was used to determine the factors associated with hs-cTnT.

Results: The characteristics of the PsA cohort are presented in table 1. Remarkably, in the majority of patients, both skin and joint disease were well controlled. PsA and TAMCIS cohorts had a similar range of age (51.5 vs $48 \mathrm{yr}$ ) but different gender representation: $47 \%$ vs $72.5 \%$ of males $(p<0.001)$. PsA exhibited a higher prevalence of traditional CVD risk factors compared to the TAMCIS cohort: BMI 28 vs 26.5 ( $p=0.002)$, current smokers $20.8 \%$ vs $10.1 \%$ ( $p=0.002)$, hypertension $25 \%$ vs $15 \%(p=0.007)$, dyslipidemia prevalence $34 \%$ vs $27 \%(p=0.101)$, diabetes $19.8 \%$ vs $4.6 \%(p<0.0001)$. Due to these differences, a matched control group was used for comparison of troponin. Detectable hs-cTnT was present in $29.5 \%$ of the PsA patients compared to $19.3 \%$ in the controls. $(p=0.114)$ Factors associated with detectable hs-cTnT in PsA were consistent with traditional factors and included male gender $(p=0.007)$, age $(p=0.005)$, hypertension $(p<0.001)$, and $D M$ $(p<0.001)$. No correlation between detectable hs-cTnT level and psoriasis/PsA duration, disease severity, treatment with DMARDs or biologics was found.
Table 1 Characteristics of the PsA cohort $(n=96)$

\begin{tabular}{ll}
\hline Gender, \% males & $47 \%$ \\
\hline Age, years & $51.5 \pm 13$ \\
Psoriasis duration & $17 \pm 13$ \\
Psoriasis activity: remission/mild/moderate to severe & $34 \% / 56 \% / 10 \%$ \\
PsA duration & $9.4 \pm 8.1$ \\
PsA activity: remission/low/moderate/severe & $37 \% / 33 \% / 22 \% / 8 \%$ \\
Concomitant sDMARDs treatment (\%) & $49 \%$ \\
Concomitant biologic treatment (\%) & $65 \%$ \\
Biologic treatment exposure (past or present) (\%) & $71.8 \%$
\end{tabular}

Conclusions: This is the first study to report a detectable hs-cTnT level in up to $30 \%$ in patients with well controlled PsA, asymptomatic for CVD, warranting a special attention to monitoring CVD risk factors and manifestations in this group. Traditional CVD risk factors but not measures of disease activity were associated with detectable hs-cTnT. The latter may be explained by a potential positive impact of anti-rheumatic therapies on the cardiovascular profile. Further prospective studies addressing the predictive role of hs-cTnT for CVD events in PsA are needed.

\section{REFERENCES:}

1. Saunders JT, et al. Circulation 2011;123

2. Ogdie A, et al. Ann Rheum Dis 2015;74.

Disclosure of Interest: None declared DOI: 10.1136/annrheumdis-2018-eular.4002

\section{FRI0680 ON BLOOD LEUCOCYTES CAN CAPTURE INTERFERON STATUS AND CORRELATES WITH USTEKINUMAB (STELARA) THERAPY RESPONSE IN PSORIATIC DISEASE.}

Y. M. El-Sherbiny ${ }^{1}$, L. Savage ${ }^{1}$, A. Alase ${ }^{1}$, E. Vital ${ }^{1}$, M. Wittmann ${ }^{1}$, P. Emery ${ }^{1}$, D. McGonagle'. ${ }^{1}$ Leeds Institute of Rheumatic and Musculoskeletal Medicine, University Of Leeds, Leeds, United Kingdom

Background: Recently Ustekinumab, an IL12/23 p40 monoclonal antibody that is licensed for Psoriatic Arthritis and Psoriasis, showed promising results in phase II trials in SLE- a prototype IFN mediated disease. We previously confirmed a novel IFN gene scoring system associated with the different SLE symptoms (manuscript in review). Additionally, we also recently validated in two independent cohorts the value of BST2/ tetherin as IFN-I biomarker assay correlates with clinical activity and predicts clinical flare in SLE. Given that psoriasis has several SNPs in the IFN pathway; Thus mechanistic studies of the effect of Ustekinumab on the IFN pathway can be explored in this disease setting.

Objectives: This work tested the hypothesis that a novel interferon type I (IFN-I) status markers in the blood and skin of Ustekinumab treated psoriasis patients might correlate with therapy responses and provide insights into how $\mathrm{p} 40$ blockers may affect IFN pathways in a relevant human disease model.

Methods: Skin biopsies and peripheral blood at baseline (before therapy 24weeks, 54 weeks) from 23 Ustekinumab patients with psoriasis who had ultrasound imaging confirmed subclinical enthesopathy) were recruited. Cellular immunophenotyping was performed using multi-parameter flow cytometry to detect tetherin on (Monocyte, B cells, T cells and neutrophils). All data was compared to age-matched samples from healthy controls $(\mathrm{HC})$. Skin biopsies were digested and RNA extracted, qPCR of common ISGs genes were analysed in lesional and peri-lesional at corresponding time points.

Results: Tetherin showed a higher level of expression on blood subsets of psoriasis compared to $\mathrm{HC}$ at baseline on Monocytes, T cells, NK cells and B cells $(p=0.003,=0.005,=0.035,=0.002)$ compared to the baseline. No significant changes observed between baseline and 2 nd visit 24 weeks. Interestingly, a substantial reduction in tetherin expression at (52 weeks) in psoriasis was observed in all subsets in Monocytes, T cells, NK cells and B cells (all $p<0.0001$ ) correlating with patient response to therapy. IFN signature by TaqMan revealed higher expression in skin biopsies distinctive ISGs compared to HC, e.g. IFI27, STAT1, IFI16 and IRF7 all corrected post-Ustekinumab therapy to normal levels. Paired analysis revealed a stronger IFN signature in lesional biopsies vs non-lesional biopsies, e.g. IFI27 ( $p=0.0312$, Wilcoxon matched pair-rank test). 